\title{
Mononucleotide repeats are asymmetrically distributed in fungal
} genes

\author{
Mark WJ van Passel* and Leo H de Graaff
}

\author{
Address: Laboratory of Microbiology, Wageningen University, Dreijenplein 10, 6703 HB, Wageningen, the Netherlands \\ Email: MarkWJ van Passel* - Mark.vanPassel@wur.nl; Leo H de Graaff - Leo.deGraaff@wur.nl \\ * Corresponding author
}

Published: II December 2008

BMC Genomics 2008, 9:596 doi:10.1 |86/147|-2/64-9-596
Received: 24 September 2008

Accepted: II December 2008

This article is available from: http://www.biomedcentral.com/I47I-2/64/9/596

(C) 2008 van Passel and de Graaff; licensee BioMed Central Ltd.

This is an Open Access article distributed under the terms of the Creative Commons Attribution License (http://creativecommons.org/licenses/by/2.0), which permits unrestricted use, distribution, and reproduction in any medium, provided the original work is properly cited.

\begin{abstract}
Background: Systematic analyses of sequence features have resulted in a better characterisation of the organisation of the genome. A previous study in prokaryotes on the distribution of sequence repeats, which are notoriously variable and can disrupt the reading frame in genes, showed that these motifs are skewed towards gene termini, specifically the 5 ' end of genes. For eukaryotes no such intragenic analysis has been performed, though this could indicate the pervasiveness of this distribution bias, thereby helping to expose the selective pressures causing it.

Results: In fungal gene repertoires we find a similar 5 ' bias of intragenic mononucleotide repeats, most notably for Candida spp., whereas e.g. Coccidioides spp. display no such bias. With increasing repeat length, ever larger discrepancies are observed in genome repertoire fractions containing such repeats, with up to an 80 -fold difference in gene fractions at repeat lengths of $10 \mathrm{bp}$ and longer. This species-specific difference in gene fractions containing large repeats could be attributed to variations in intragenic repeat tolerance. Furthermore, long transcripts experience an even more prominent bias towards the gene termini, with possibly a more adaptive role for repeat-containing short transcripts.
\end{abstract}

Conclusion: Mononucleotide repeats are intragenically biased in numerous fungal genomes, similar to earlier studies on prokaryotes, indicative of a similar selective pressure in gene organization.

\section{Background}

Genetic patterns could not be studied comprehensively until whole genome sequences became available. The first such genome-wide sequence analysis focused on both the abundance and distribution of competence-associated sequence motifs in the prokaryote Haemophilus influenzae [1]. Since then, as more genome sequences became available, numerous other genetic features were studied in prokaryotes [2-4] and eukaryotes [5,6]. Many such genome-wide analyses concentrated on the genomic distribution of simple sequence repeats (SSRs, also known as microsatellites), stretches of mono- and oligonucleotide repeats [7-10].

SSRs generally occur more frequently in non-coding regions of the genome. One of the reasons for this avoidance in the protein coding regions is that many SSRs predispose for disruptive frameshifts via strand-slippage during replication, transcription or translation $[8,11]$. Recently Ackermann and Chao postulated that selection for sequence stability in coding regions has been a pervasive force in the distribution biases of mononucleotide repeats (MNRs) in both prokaryotic and eukaryotic 
genome sequences [12]. More recently, we discovered that MNRs also display a biased distribution within coding sequences: in a wide range of both bacterial and archaeal genomes, mononucleotide repeats were predominantly biased towards the 5' end of genes [13], presumably to prevent or reduce the expression of toxic or costly frameshifted proteins. However, for eukaryotes no such intragenic distribution analysis has been carried out.

The Fungi represent a kingdom within the eukaryotic domain with many fully sequenced representatives. These can vary in genome size (less than $3 \mathrm{Mbp}$ for Encephalitozoon cuniculi, up to an estimated 82 Mbp for Puccinia graminis), cellular organization (single cells or multicellular organisms) as well as life-style (saprophytic or pathogenic) $[14,15]$. With respect to SSRs, a previous analysis in sequenced fungal genomes described different patterns of their occurrence [9], but did not study their intragenic distribution.

Here we analyze genome-wide sets of predicted coding sequences from fully sequenced fungal genomes, and assess the intragenic distribution patterns of mononucleotide repeats. MNRs are more commonly present than the more complex repeats, which makes their interspecific distribution comparisons possible. This could illuminate the similarities and differences between the distributions of disruptive repeats in prokaryotes and eukaryotes, and help identify the selective forces that brought about these biases.

\section{Methods}

Transcript data of the coding regions (excluding introns) from 47 fungal genomes were obtained from the Broad Institute http://www.broad.mit.edu/annotation/. The datasets, their size and the description of the strains are given in Table 1. Truncated genes, transcripts with annotated internal stop codons and genes that are not multiples of three in length were excluded from the analyses, which basically rely on a genome-wide quantitative assessment of MNRs in the five quintiles of the annotated protein-coding sequences. Sequence motif analyses and codon usage profiles were carried out using in-house perl scripts, which are available upon request.

Fungal transcripts were compared to the KOG database [16] according to the nearest-neighbor method (using raw scores) [17] in order to distinguish between transcripts with a well-defined ortholog and transcripts that lacked an ortholog in the database.

\section{Results Intragenic distribution biases of mononucleotide repeats in fungal coding sequences}

For each genome we tested the intragenic distribution profiles of the longest MNRs in the five quintiles of all pre- dicted protein coding genes, with a minimal occurrence of 100 repeats of that length in the genomic transcript data (see Figure 1 for 6 representative cases, and Additional File 1 for all repeat distributions). The gene repertoires of most strains (25/47 genomes) show a non-proportional distribution of MNRs over the five quintiles (Chi-square, 4 degrees of freedom, $\mathrm{p}<0.05$, Additional File 1). The strongest bias is observed in Candida parapsilosis, where $83 \%$ (104/125 repeats) of all repeats of 10 bp or longer are in the first quintile of the genes. In several cases, the majority of repeats are in the last quintile (80-100\%) of the genomic gene set (e.g., Botrytis cinerea and Chaetomium globosum).

\section{Genes with mononucleotide repeats of 15 bp or longer}

The 294 fungal transcripts with repeats of 15 residues or longer from all genomes combined are given in Additional File 2. Again, when analyzing the intragenic distribution of the 298 repeats in these 294 fungal genes, a strong bias towards the first quintile of the genes is observed, both in transcripts with well-described functional orthologs (KOGs), but also in transcripts without orthologs (nKOGs) in the KOG database (Figure 2). As for the extreme cases, Aspergillus fumigatus, Aspergillus niger, Aspergillus terreus, Candida lusitaniae, Histoplasma capsulatum, Paracoccidioides brasiliensis and Rhizopus oryzae each contain genes with mononucleotide repeats over 30 nucleotides long. The gene with the longest repeat is encountered in A. terreus (transcript ATET_00185), which contains a stretch of 68 consecutive adenine residues, encoding 22 consecutive lysines. Among the intragenic repeats of over 15 nucleotides in length, guanine and cytosine tracts are relatively rare with only 80 out of 298 repeats, similar as to what was found previously [18]. Still, some genomes harbour transcripts with repeats that consist solely of long guanine or cytosine tracts; Chaetomium globosum (6 genes), Coprinus cinereus (4 genes), Neurospora crassa (4 genes) and Sclerotinia sclerotiorum (5 genes). Other genomes have repeat-containing genes with only adenine or thymine tracts of over 15 residues in length: Candida albicans wo1 (11 genes), Candida lusitaniae (13 genes), C. tropicalis (41 genes), Coccidioides immitis rs (8 genes) and $R$. oryzae (11 genes).

\section{Trinucleotide repeats in Neurospora crassa coding regions}

Previous analyses have shown that fungal genomes also harbour oligonucleotide repeats, which on occasion have been associated with particular processes [19]. These repeats are mostly typical for the individual species, and though abundant, the total repeat-specific counts are still often less than 100 in the coding regions of a genome. However, in $N$. crassa, numerous trinucleotide repeats have been identified [20], of which only a few are encountered in large numbers in the coding regions: (GGT) $)_{n}(117$ repeats of $n>4)$, (TTG $)_{n}(144$ repeats of $n>2)$ and $(\text { ACA })_{n}$ 
Table I: List of the 47 analyzed species, the number of coding sequences and their scientific, industrial or biomedical merit.

\begin{tabular}{|c|c|c|}
\hline Name & CDS* & Description of strain relevance \\
\hline Aspergillus clavatus & 9110 & Animal pathogen \\
\hline Aspergillus flavus & 12434 & Phytopathogen \\
\hline Aspergillus fumigatus & 9884 & Opportunistic human pathogen \\
\hline Aspergillus nidulans & 10474 & Model organism \\
\hline Aspergillus niger & 6329 & Industrial strain \\
\hline Aspergillus oryzae & 12063 & Industrial strain \\
\hline Aspergillus terreus & 10400 & Opportunistic human pathogen/industrial strain \\
\hline Batrachochytrium dendrobatidis & 8779 & Amphibian pathogen \\
\hline Botrytis cinerea & 15512 & Phytopathogenic fungus \\
\hline Candida albicans sc53/4 & 5916 & Opportunistic human pathogen \\
\hline Candida albicans wol & 5851 & Opportunistic human pathogen \\
\hline Candida guilliermondii & 5897 & Haploid relative of Candida albicans \\
\hline Candida lusitaniae & 5891 & Opportunistic human pathogen \\
\hline Candida parapsilosis & 5687 & Opportunistic human pathogen \\
\hline Candida tropicalis & 6216 & Opportunistic human pathogen \\
\hline Chaetomium globosum & 10987 & Important decomposers of biomass \\
\hline Coccidioides immitis h538.4 & 10480 & Human pathogen \\
\hline Coccidioides immitis rmscc 2394 & 10368 & Human pathogen \\
\hline Coccidioides immitis rmscc 3703 & 10379 & Human pathogen \\
\hline Coccidioides immitis rs & 10609 & Human pathogen \\
\hline Coccidioides posadasii rmscc 3488 & 9932 & Human pathogen \\
\hline Coccidioides posadasii str. silveira & 10070 & Human pathogen \\
\hline Coprinus cinereus & 13523 & Model organism for multicellularity \\
\hline Cryptococcus neoformans h99 & 7077 & Human pathogen \\
\hline Debaryomyces hansenii & 6101 & Cryo- and halotolerant marine yeast \\
\hline Fusarium graminearum & 13220 & Representative of an important family of phytopathogens \\
\hline Fusarium oxysporum $f$. sp. lycopersici & 17202 & Phytopathogen and model organism for evolutionary research \\
\hline Fusarium verticillioides & 14002 & Phytopathogen and model organism for evolutionary research \\
\hline Histoplasma capsulatum nam I & 9164 & Human pathogen \\
\hline Lodderomyces elongisporus & 5739 & Closest sexual relative to Candida albicans \\
\hline Magnaporthe grisea & 12564 & Phytopathogen \\
\hline Neosartorya fischeri & 10383 & Opportunistic human pathogen and involved in food spoilage \\
\hline Neurospora crassa & 9795 & Model organism \\
\hline Paracoccidioides brasiliensis pb03 & 9235 & Human pathogen \\
\hline Puccinia graminis $f . s p$. tritici & 20462 & Phytopathogen \\
\hline Pyrenophora tritici-repentis & 12092 & Phytopathogen \\
\hline Rhizopus oryzae & 17074 & Representative agent of mucormycosis \\
\hline Saccharomyces cerevisiae $r m I I-I a$ & 5272 & Model organism \\
\hline Schizosaccharomyces japonicus yfs 275 & 5122 & Model organism for comparative genomics study \\
\hline Schizosaccharomyces octosporus yfs 286 & 4906 & Model organism for comparative genomics study \\
\hline Schizosaccharomyces pombe $972 \mathrm{~h}$ & 4991 & Model organism \\
\hline Sclerotinia sclerotiorum & 13704 & Broad host range phytopathogen \\
\hline Stagonospora nodorum & 15949 & Phytopathogen \\
\hline Uncinocarpus reesii & 7777 & Closest known relative to the pathogenic Coccidioides \\
\hline Ustilago maydis & 6517 & Phytopathogen \\
\hline Verticillium albo-atrum vams. 102 & 10098 & Phytopathogen \\
\hline Verticillium dahliae vdls. I 7 & 10453 & Phytopathogen \\
\hline
\end{tabular}

*) All transcripts, except those with annotated internal stop codons, annotated as missing ends or transcripts that are not multiples of three in length.

(117 repeats of $n>7$ ). Changes in the copy number of trinucleotides in a gene do not cause a shift in the reading frame, and therefore we hypothesized that these repeats need not be intragenically biased. Nevertheless, we observed that all three trinucleotide repeats occur more frequently at the gene termini of coding regions, with over half of all GGT repeats in the last quintile of the coding regions (Figure 3 ). This suggests that the bias of repeats may not be caused solely by their risk for frameshifts. 


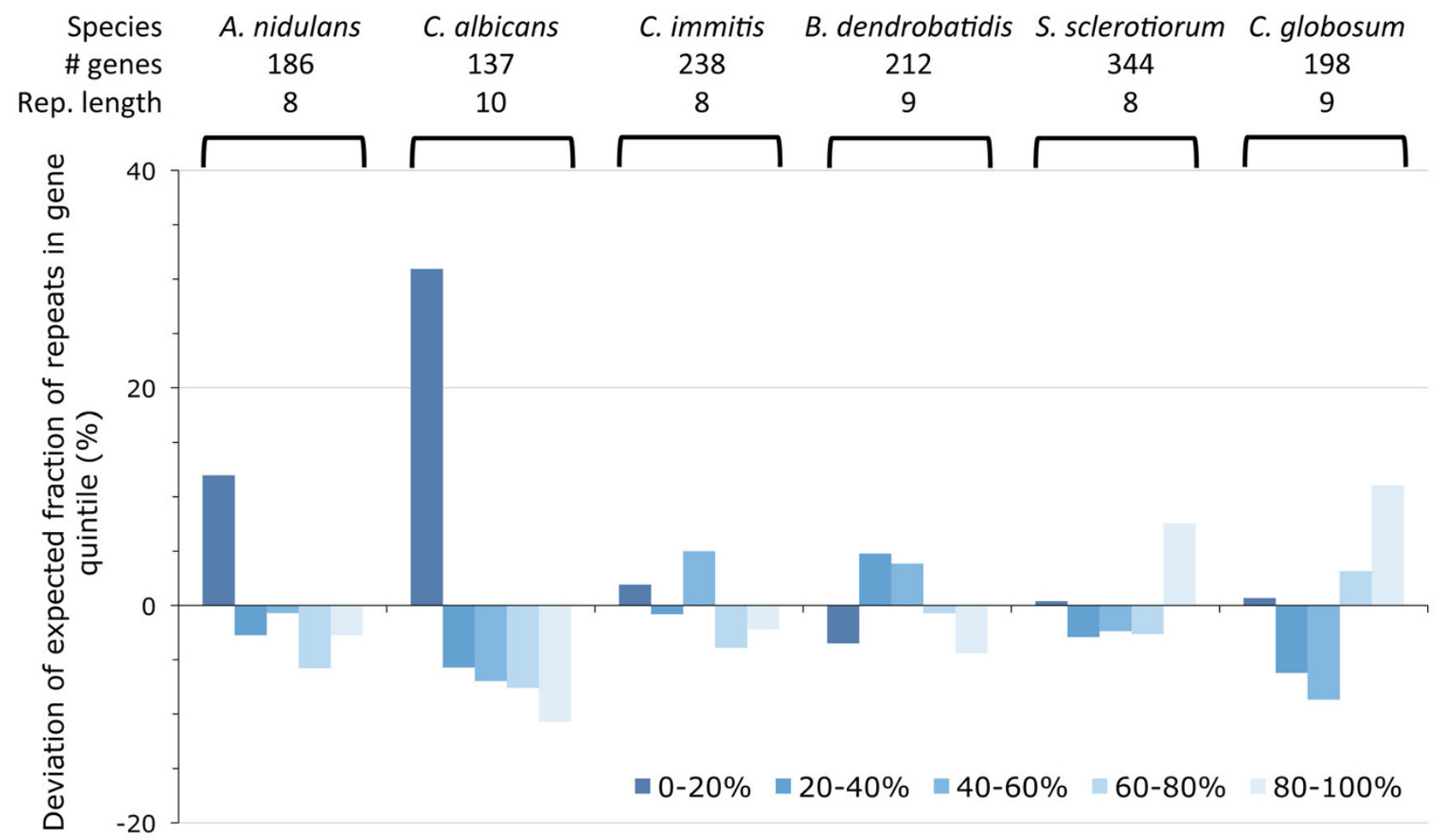

Figure I

Six examples of repeat distribution profiles in the gene quintiles of fungal gene repertoires. Represented are the deviations of the expected value per quintile (i.e., 20\%). \# genes signifies the number of genes with mononucleotide repeats (per genome), and Rep. length signifies the length of the MNR in basepairs.

\section{Intragenic repeat resistance as indicated by genome repertoire analyses and consecutive homogenous codon usage profiles}

The fractions of genes per genome that contain a mononucleotide repeat decreases rapidly with increasing repeat lengths in all tested genomes (Figure 4). Nevertheless, a substantial difference may exist between the tolerances of the species to disruptive intragenic mononucleotide repeats. Short repeats (5 bp) are encountered in most $(72-97 \%)$ of the protein-coding gene repertoires of all 47 tested species, and are never intragenically biased. However, with increasing repeat lengths, ever larger discrepancies arise between the percentages of the different genomic gene repertoires that contain repeats of such lengths (Figure 4, Additional File 3). C. tropicalis has an $80 \times$ larger gene fraction containing repeats of 10 residues or longer than Neosartorya fischeri ( $3 \%$ and $~ 0.03 \%$, respectively), although $N$. fischeri contains almost twice as many genes. This higher gene fraction with repeats suggests that $C$. tropicalis enjoys a much higher tolerance for disruptive intragenic repeats.

Comparing the expected versus observed frequencies of neighbouring lysine or phenylalanine codon, we find that
N. fischeri avoids flanking homogeneous codons (i.e., AAA-AAA-AAA for lysine, and TTT-TTT-TTT for phenylalanine) to a much higher extent than $C$. tropicalis, corroborating the higher repeat tolerance of the latter species (data not shown).

\section{Intrageneric comparisons of intragenic mononucleotide repeats}

The dataset contains the gene repertoires from different species and strains from the same genus, which allows us to test for intrageneric heterogeneity of repeat distribution biases. Three genera are tested, Aspergillus, Candida and Coccidioides. Firstly, both Aspergillus and Candida species show a 5' bias for intragenic repeat distribution patterns, which are relatively mild in Aspergillus, and strong in Candida. The sole outlier in the Aspergilli is Aspergillus flavus, which contrary to its relatives shows no (strong) $5^{\prime}$ bias. In Candida, there is a very strong bias of repeats to the 5 ' end, except for the species Debaryomyces hansenii, which shows a 3' bias. The two species with the lowest observed 5' bias are Candida guilliermondii and C. lusitaniae, which are closely related species, and both branch off with Debaromyces hansenii in one of the so-called CTGsubclades [21]. Finally, none of the two tested Coccidio- 


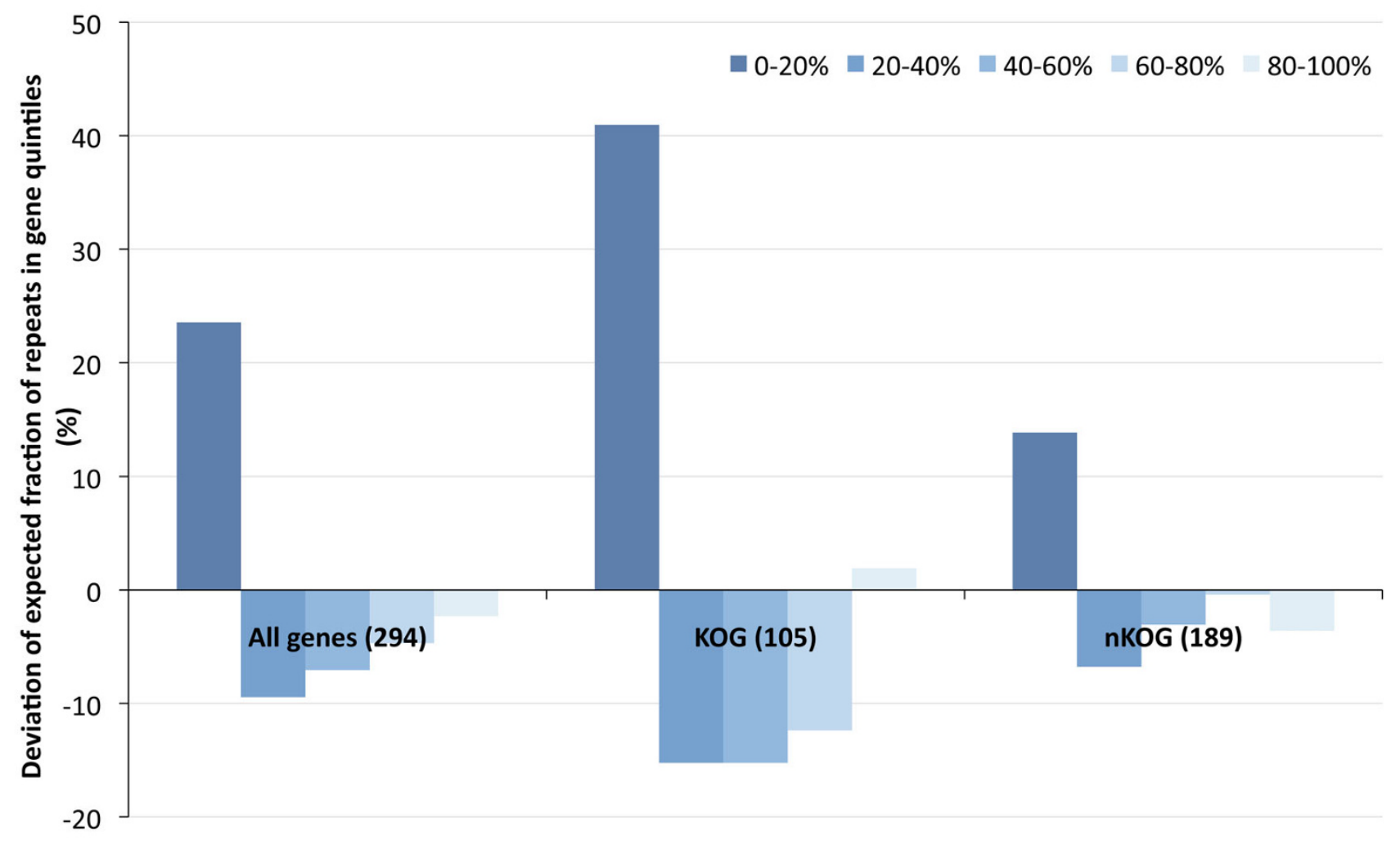

Figure 2

The distribution of very long repeats (> I 5 bp mononucleotide repeats, 298 repeats, 294 genes) in the quintiles of the predicted coding regions from all tested fungal genomes. Represented are the deviations of the expected value per quintile (i.e., 20\%).

ides species (eight strains) show a distribution bias in mononucleotide repeats.

\section{Authenticity of the intragenic repeat bias}

Of the 294 fungal transcripts that contain repeats of $15 \mathrm{bp}$ or longer, $36 \%(105 / 294)$ could be assigned to a eukaryotic cluster of orthologous genes (KOG, Additional File 2, [16]). In this set of 105 orthologs to bona fide protein-coding genes, we observe a significant distribution bias of the repeats towards the 5 ' end (61\% of all repeats are present in the first quintile of these 105 genes, $\mathrm{p}<0.05$, Figure $5 \mathrm{~A})$. Moreover, we discern that the genes with repeats at the gene termini are on average much longer than genes with the repeat in the middle of the gene (Figure 5B). A similar trend was observed for the fungal genes that could not be assigned to a KOG (termed nKOG), i.e., 34\% of all repeats are in the first gene quintile, and transcripts with repeats at the gene termini are longer than transcripts with a repeat in the middle of the gene (Figure 5A and 5B). Interestingly, a large proportion of the 105 genes with long repeats that also have homologs in the KOG database are found in the pathogenic species $C$. tropicalis and
C. parapsilosis (48 genes, 47 (98\%) of which have the long repeat in the first gene quintile).

\section{Discussion and conclusion}

Analyses of prokaryotic gene repertoires revealed a persistent bias of disruptive sequence repeats towards gene termini, potentially to curtail the metabolic costs or toxicity associated with transcribing and (or) translating nonfunctional genes [13]. In order to explore this phenomenon for the eukaryotic domain, we investigated the genome repertoires from 47 sequenced fungi, and discovered that this pattern is also evident in the fungal kingdom.

Still, large discrepancies exists between the intragenic distribution bias of MNRs of the different genomes: Coccidioides spp. show no intragenic preference for sequence repeats, whereas the Candida spp. demonstrate a very strong 5 ' end bias, with even up to $83 \%$ of all intragenic repeats in $C$. parapsilosis in the first gene quintile. Some genomes display a strong $3^{\prime}$ end bias for intragenic repeats, such as Botrytis cinerea, which has $36 \%$ of its 


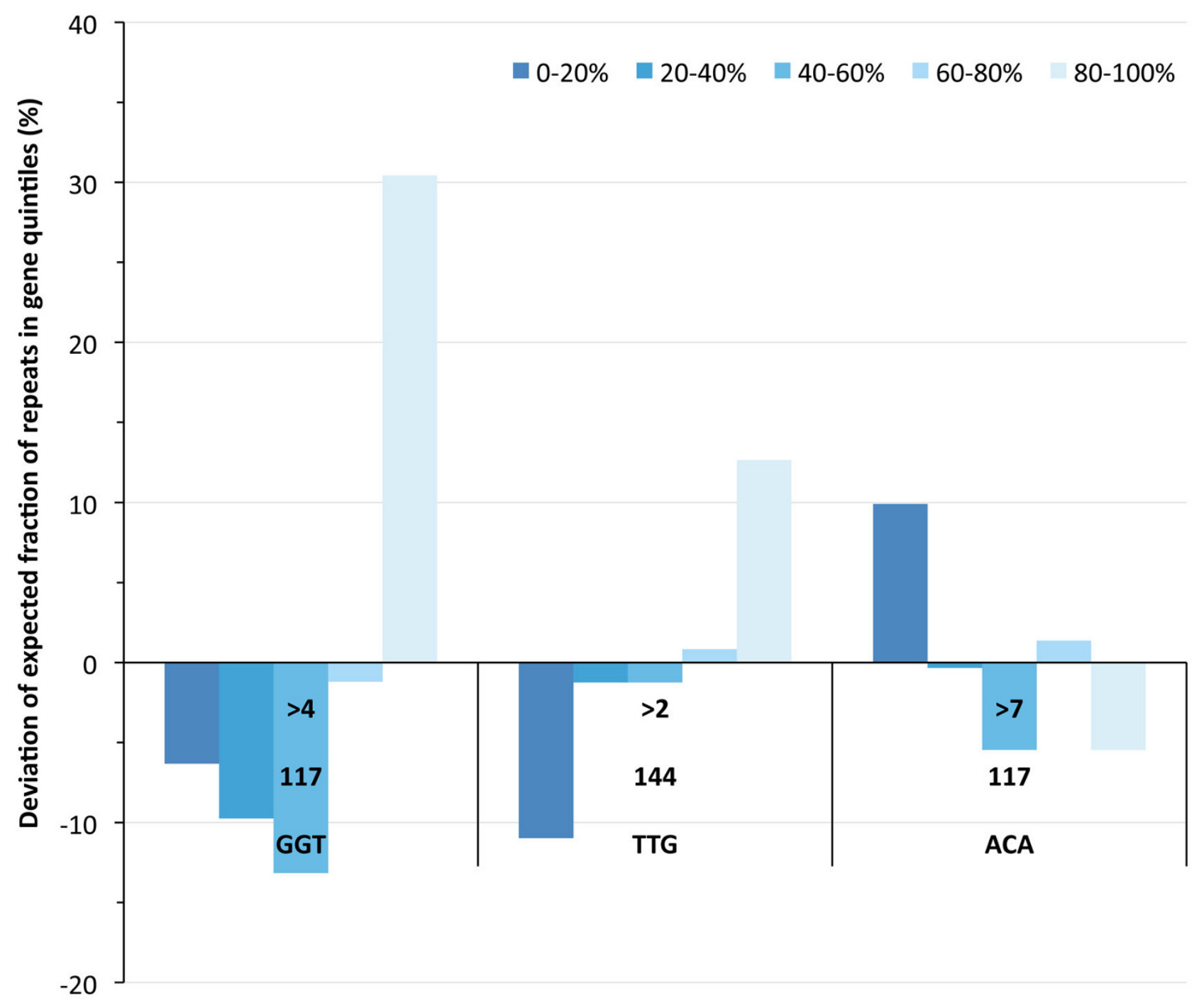

Figure 3

Distribution of trinucleotide repeats GGT, TTG and ACA in the quintiles of the protein coding genes from Neurospora crassa. Represented are the deviations of the expected value (i.e., $20 \%$ ).

mononucleotide repeats in the last gene quintile. A bias of these disruptive repeats to either gene terminus agrees with a selection pressure to remove potential toxic side products, or alternatively to reduce metabolic costs, similar to what was found by Akashi and Gojobori in a comparison of 'expensive' and 'cheap' amino acid usage in highly expressed genes [22].

The species-specific gene fractions that contain long repeats could be a proxy for the species' tolerance for disruptive repeats. These differences are substantial, as the gene fraction of $C$. tropicalis with repeats of ten nucleotides or longer is 80 times higher than the gene fraction in $N$. fischeri. The adjacent homogeneous codon usage in these species also shows a higher avoidance of contiguous AAA and TTT codons in the latter species. A mechanistic explanation for a higher repeat-tolerance is still unknown, but could be studied by a more detailed functional characterisation of the genes that contain these long repeats.

When analysing the most abundant intragenic trinucleotide repeats in N. crassa, we find a strong bias of these 


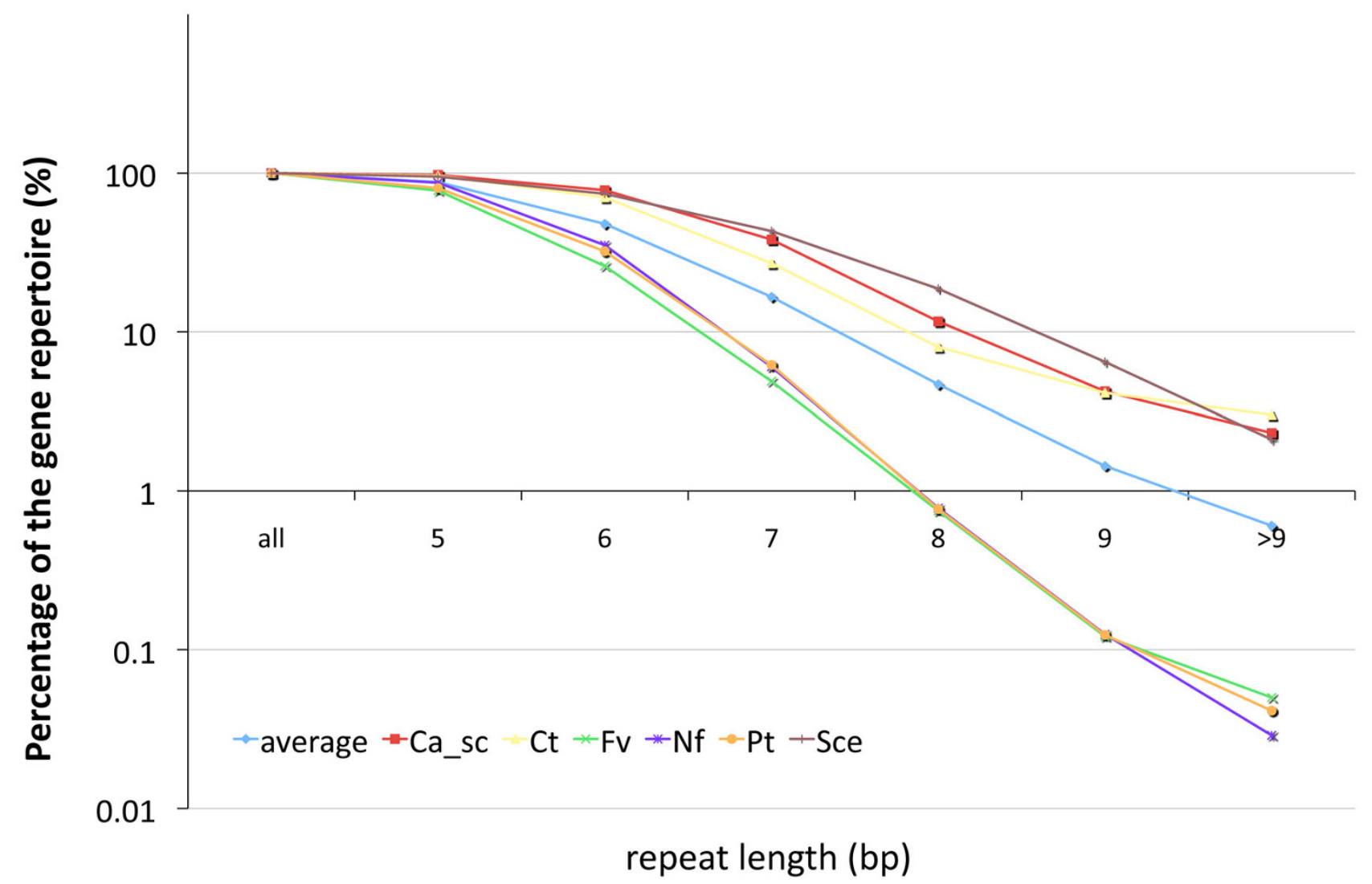

Figure 4

Percentages of the gene repertoires that contain homopolymeric tracts in fungal species: only the three species with the highest (C. albicans sc53 I4, C. tropicalis and S. cerevisiae) and three lowest (F. verticillioides, $N$. fischeri and $P$. tritici-repentis) genome fraction that contain homopolymeric tracts of said length (x-axis) are depicted, as well as the average of all 47 strains. Note the logarithmic scale on the y-axis. The data for all 47 strains is available in Additional File 3.

repeats to the gene termini, even though differences in trinucleotide repeats do not cause a shift in the reading frame. This suggests that a selection pressure to remove potential toxic side products, or alternatively to reduce metabolic costs may not be the only explanation for the bias of repeats to gene termini. Previous analyses on amino acid repeats in Drosophila spp. also showed an avoidance of these repeats in the middle of genes [23]. Numerous trinucleotide repeat disorders are known to cause disease in humans [24,25], and studies into the intragenic repeat location biases could help determine mechanistic aspects of repeat expansions and contractions.

Sequence repeats have been thought to convey adaptive benefits due to their potential to facilitate rapid changes in the coding content [26], while one study also suggested that repeats are preferentially located towards recombination hot spots [27]. We find that larger genes have a more prominent bias of repeats at their gene termini. This could mean that many of the smaller transcripts with their mononucleotide repeat in the middle are misannotations, or gene remnants after the erosion of repeat-containing genes. Alternatively, these small repeat containing genes could represent foci of genetic novelty.

This focus on intragenic distribution biases is new $[13,28]$, and could be expanded to other features previously not analysed as such, like methylation patterns, or targets for transcriptional or translational regulation. This can help resolve the origin and organization of new genetic features, as well as the evolutionary forces governing them.

\section{Authors' contributions}

MWJvP conceived the project, carried out the analyses, interpreted the results and wrote the paper. LHdG inter- 

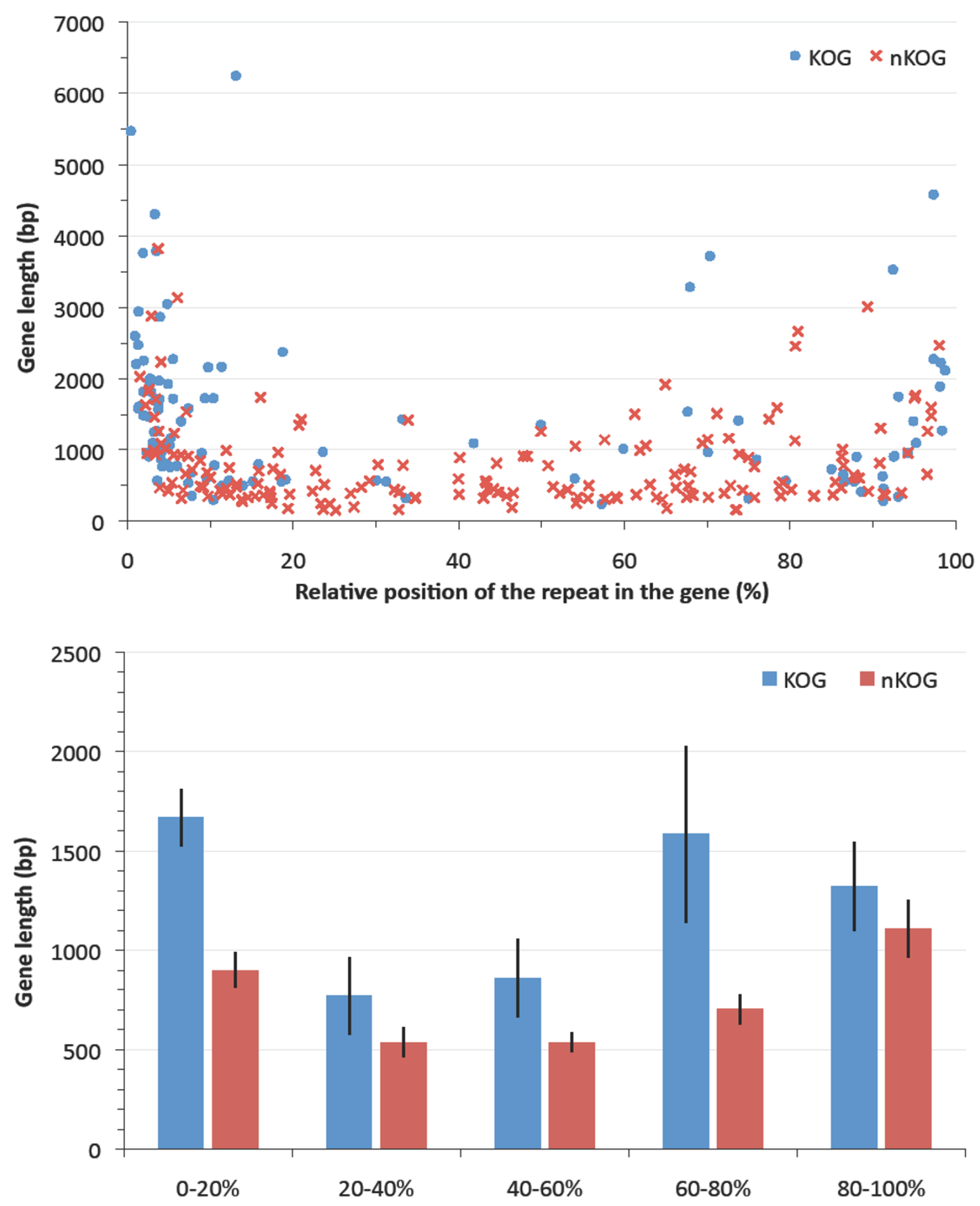

\section{Figure 5}

A) Distribution of the relative position of long repeats ( 15 residues or longer) in a gene vs. the gene length. Genes with a functional KOG annotation (KOG) and those without such an annotation (nKOG) are depicted with blue circles and red crosses, respectively. B) The average lengths of the transcripts (and the standard error of the means) are depicted for genes with a KOG annotation (blue) and genes without a KOG annotation (red), with respect to the position of the repeat in that transcript. 
preted the results and wrote the paper. Both authors read and approved the final manuscript.

\section{Additional material}

\section{Additional File 1}

Total counts of repeats in the gene quintiles. Total counts of repeats in the gene quintiles (the columns numbered 1 to 5 correspond to the first, second, third, fourth and fifth quintile of the gene), the fractions of the repeat counts in the different quintiles and the deviation from the expectancy values (i.e., 20\%).

Click here for file

[http://www.biomedcentral.com/content/supplementary/1471-

2164-9-596-S1.docx]

\section{Additional File 2}

Gene lists of the fungal species that have intragenic repeats $15 \mathrm{bp}$ or longer. Gene lists of the fungal species that have intragenic repeats $15 \mathrm{bp}$ or longer (Aspergillus flavus, Batrachochytrium dendrobatidis, Candida guilliermondii, Debaryomyces hansenii, Pyrenophora triticirepentis and Schizosaccharomyces japonicus yfs 275 do not contain predicted genes with such long repeats). Since some genomes do not have unique gene identifiers, their names are complemented with the line number of the gene name in the original fasta file. Consecutive entries highlighted in green signify identical genes (a total of 4 are identified). Click here for file

[http://www.biomedcentral.com/content/supplementary/14712164-9-596-S2.docx]

\section{Additional File 3}

Repertoire sizes of genes with different repeat lengths. Gene repertoire sizes of the fungi, and the counts and fractions of genes that contain repeats of lengths five until greater than nine.

Click here for file

[http://www.biomedcentral.com/content/supplementary/14712164-9-596-S3.docx]

\section{Acknowledgements}

The authors would like to thank the Broad Institute for releasing the data of the fungal genome sequencing projects. Furthermore, the authors would like to thank Carlos Echavarri-Erasun and Teun Boekhout for earlier discussions on this project, and Arnold Kuzniar for assistance with the functional classifications.

\section{References}

I. Smith HO, Tomb JF, Dougherty BA, Fleischmann RD, Venter JC: Frequency and distribution of DNA uptake signal sequences in the Haemophilus influenzae Rd genome. Science 1995, 269(5223):538-540.

2. Lawrence JG, Hendrickson H: Lateral gene transfer: when will adolescence end? Mol Microbiol 2003, 50(3):739-749.

3. Lawrence JG, Hendrickson H: Genome evolution in bacteria: order beneath chaos. Curr Opin Microbiol 2005, 8(5):572-578.

4. Karlin S, Campbell AM, Mrazek J: Comparative DNA analysis across diverse genomes. Annu Rev Genet 1998, 32: 185-225.

5. Gentles AJ, Karlin S: Genome-scale compositional comparisons in eukaryotes. Genome Res 200 I, I I(4):540-546.

6. Bussemaker HJ, Li H, Siggia ED: Regulatory element detection using a probabilistic segmentation model. Proc Int Conf Intell Syst Mol Biol 2000, 8:67-74.

7. Mrazek J, Guo X, Shah A: Simple sequence repeats in prokaryotic genomes. Proc Natl Acad Sci U S A 2007, 104(20):8472-8477.
8. van Belkum A, Scherer S, van Alphen L, Verbrugh $H$ : Shortsequence DNA repeats in prokaryotic genomes. Microbiol Mol Biol Rev 1998, 62(2):275-293.

9. Karaoglu H, Lee CM, Meyer W: Survey of simple sequence repeats in completed fungal genomes. Mol Biol Evol 2005, 22(3):639-649.

10. Coenye T, Vandamme P: Characterization of mononucleotide repeats in sequenced prokaryotic genomes. DNA Res 2005, I 2(4):22I-233.

II. Hancock JM: The contribution of slippage-like processes to genome evolution. J Mol Evol 1995, 4 I (6): 1038- 1047.

12. Ackermann $M$, Chao L: DNA sequences shaped by selection for stability. PLoS Genet 2006, 2(2):e22.

13. van Passel MW, Ochman H: Selection on the genic location of disruptive elements. Trends Genet 2007, 23(I 2):60 I-604.

14. NCBI: National Center for Biotechnology Information. 2008 [http://www.ncbi.nlm.nih.gov/genomes/leuks.cgi].

15. James TY, Kauff F, Schoch CL, Matheny PB, Hofstetter V, Cox CJ, Celio G, Gueidan C, Fraker E, Miadlikowska J, et al.: Reconstructing the early evolution of Fungi using a six-gene phylogeny. Nature 2006, 443(7 I I3):8I8-822.

16. Tatusov RL, Fedorova ND, Jackson JD, Jacobs AR, Kiryutin B, Koonin EV, Krylov DM, Mazumder R, Mekhedov SL, Nikolskaya AN, et al.: The COG database: an updated version includes eukaryotes. BMC Bioinformatics 2003, 4:4I.

17. Kuzniar A, van Ham RC, Pongor S, Leunissen JA: The quest for orthologs: finding the corresponding gene across genomes. Trends Genet 2008, 24(II):539-55I.

18. Toth G, Gaspari Z, Jurka J: Microsatellites in different eukaryotic genomes: survey and analysis. Genome Res 2000, 10(7):967-98I.

19. Malpertuy A, Dujon B, Richard GF: Analysis of microsatellites in 13 hemiascomycetous yeast species: mechanisms involved in genome dynamics. J Mol Evol 2003, 56(6):730-74I.

20. Kim TS, Booth JG, Gauch HG Jr, Sun Q, Park J, Lee YH, Lee K: Simple sequence repeats in Neurospora crassa: distribution, polymorphism and evolutionary inference. BMC Genomics 2008, 9:31.

21. Fitzpatrick DA, Logue ME, Stajich JE, Butler G: A fungal phylogeny based on $\mathbf{4 2}$ complete genomes derived from supertree and combined gene analysis. BMC Evol Biol 2006, 6:99.

22. Akashi H, Gojobori T: Metabolic efficiency and amino acid composition in the proteomes of Escherichia coli and Bacillus subtilis. Proc Natl Acad Sci USA 2002, 99(6):3695-3700.

23. Huntley MA, Clark AG: Evolutionary analysis of amino acid repeats across the genomes of 12 Drosophila species. Mol Biol Evol 2007, 24( ( 2):2598-2609.

24. Mirkin SM: Expandable DNA repeats and human disease. Nature 2007, 447(7 I 47):932-940.

25. Kovtun IV, McMurray CT: Features of trinucleotide repeat instability in vivo. Cell Res 2008, I8(I): 198-2/3.

26. Kashi $Y$, King DG: Simple sequence repeats as advantageous mutators in evolution. Trends Genet 2006, 22(5):253-259.

27. Bagshaw AT, Pitt JP, Gemmell NJ: High frequency of microsatellites in S. cerevisiae meiotic recombination hotspots. $B M C$ Genomics 2008, 9:49.

28. van Passel MW: An intragenic distribution bias of DNA uptake sequences in Pasteurellaceae and Neisseriae. Biol Direct 2008, 3:12. 